\title{
Hyperosmotic stress induces cell cycle arrest in retinal pigmented epithelial cells
}

\author{
T Arsenijevic ${ }^{1}$, A Vujovic ${ }^{1}, \mathrm{~F}$ Libert $^{2}$, A Op de Beeck ${ }^{3}$, A Hébrant ${ }^{2}$, S Janssens ${ }^{1,4}, \mathrm{~F}$ Grégoire $^{1}$, A Lefort ${ }^{2}, \mathrm{~N}$ Bolaky ${ }^{1}$, \\ J Perret ${ }^{1}$, L Caspers ${ }^{4}, \mathrm{~F}$ Willermain $^{2,4}$ and C Delporte*,
}

Osmotic changes occur in many tissues and profoundly influence cell function. Herein, we investigated the effect of hyperosmotic stress on retinal pigmented epithelial (RPE) cells using a microarray approach. Upon 4-h exposure to $100 \mathrm{mM} \mathrm{NaCl}$ or $200 \mathrm{mM}$ sucrose, 79 genes were downregulated and 72 upregulated. Three gene ontology categories were significantly modulated: cell proliferation, transcription from RNA polymerase II promoter and response to abiotic stimulus. Fluorescentactivated cell sorting analysis further demonstrated that owing to hyperosmotic stimulation for $24 \mathrm{~h}$, cell count and cell proliferation, as well as the percentage of cells in G0/G1 and S phases were significantly decreased, whereas the percentage of cells in $\mathrm{G}_{2} / \mathrm{M}$ phases increased, and apoptosis and necrosis remained unaffected. Accordingly, hyperosmotic conditions induced a decrease of cyclin B1 and D1 expression, and an activation of the p38 mitogen-activated protein kinase. In conclusion, our results demonstrate that hypertonic conditions profoundly affect RPE cell gene transcription regulating cell proliferation by downregulation cyclin D1 and cyclin B1 protein expression.

Cell Death and Disease (2013) 4, e662; doi:10.1038/cddis.2013.189; published online 6 June 2013

Subject Category: Experimental Medicine

Consequences of osmostress have been extensively studied, mostly in renal cells that can be exposed to very high concentrations of $\mathrm{NaCl}$ and urea. Hyperosmotic stress classically induces cytoskeleton rearrangement, cell cycle arrest, with protein and DNA damage. In order to survive, cells must react through an osmoadaptative response. A prominent feature of the latter consists in an increased expression of organic osmolytes and heat shock proteins. However, it appears that osmostress modulates a very wide number of cell function through the regulation of mRNA transcription, protein translation and activity. Moreover, recent studies have found that osmotic stress response is absolutely not restricted to renal cells but on the contrary affect a wide variety of cell type. ${ }^{1,2,3}$

Retinal pigmented epithelium constitutes the most external layer of the retina. It is made of a monolayer of retinal pigmented epithelial (RPE) cells involved in a series of important retinal function such as outer segment photoreceptor phagocytosis, vitamin A cycle or excess light absorption. ${ }^{4}$ In addition, as the external part of the blood retinal barrier, it is implicated in water and osmolytes movements, as well as immune functions. ${ }^{4}$ RPE cells are thus logically involved in various retinal diseases where their phenotype is drastically changed. For example, during diabetic retinopathy, or proliferative vitreoretinopathy, RPE cells start to proliferate, form contractile membranes and contribute to the destruction of the retinal architecture. All these aspects of RPE cell biology are intensively addressed in different in vitro cell culture models. In this context, the ARPE-19 cell line are the most frequently used human RPE cell-derived cell line used to investigate the effects of multiple stimuli that are known to have a role in the pathogenesis of eye diseases.

This study was designed to identify major cellular pathway modified by hyperosmotic stress in the human RPE cell line ARPE-19.

\section{Results}

Gene expression profile in ARPE-19 cells submitted to hyperosmotic conditions. The gene expression profiles of ARPE-19 cells submitted to iso-osmotic (control condition, $\mathrm{Na0})$ and hyperosmotic conditions (100 mM NaCl (Na100), $200 \mathrm{mM}$ sucrose (Su200)) at $4 \mathrm{~h}$ were obtained using microarray technology. The number of deregulated genes resulting after appropriate normalization and filtering were 323 for Na100, with 182 downregulated and 141 upregulated; and 296 genes for Su200, with 163 downregulated and 133 upregulated. There were 151 genes similarly modified under Na100 and Su200 conditions, as shown in the Venn diagram, with 79 downregulated and 72 upregulated (Figure 1a). Functional annotation chart analysis of this subset of 151 genes was investigated using DAVID bioinformatic resources

\footnotetext{
${ }^{1}$ Laboratory of Pathophysiological and Nutritional Biochemistry, Université Libre de Bruxelles, Brussels, Belgium; ${ }^{2}$ I.R.B.H.M, Université Libre de Bruxelles, Brussels, Belgium; ${ }^{3}$ Laboratory of Virology, Université Libre de Bruxelles, Brussels, Belgium and ${ }^{4}$ Department of Ophthalmology, CHU Saint-Pierre and Brugmann, Brussels, Belgium

${ }^{*}$ Corresponding author: C Delporte, Laboratory of Pathophysiological and Nutritional Biochemistry, CP 611, Université Libre de Bruxelles, Route de Lennik 808 , Brussels B-1070, Belgium. Tel: + 3225556 210; Fax: + 3225556 230; Email: cdelport@ulb.ac.be

Keywords: hyperosmotic stress; retinal pigmented epithelial cells; cell cycle arrest

Abbreviations: BrdU, bromo-deoxyuridine; cdk, cyclin-dependent kinase; FACS, fluorescent-activated cell sorting; FDR, false discovery rate; p38, p38 mitogenactivated protein kinase; PI, propidium iodide; RPE, retinal pigmented epithelial

Received 26.3.13; revised 8.5.13; accepted 8.5.13; Edited by A Stephanou
} 


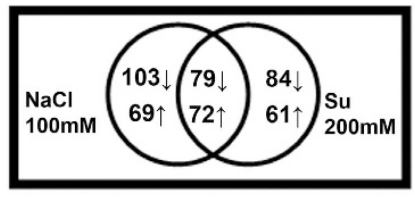

b
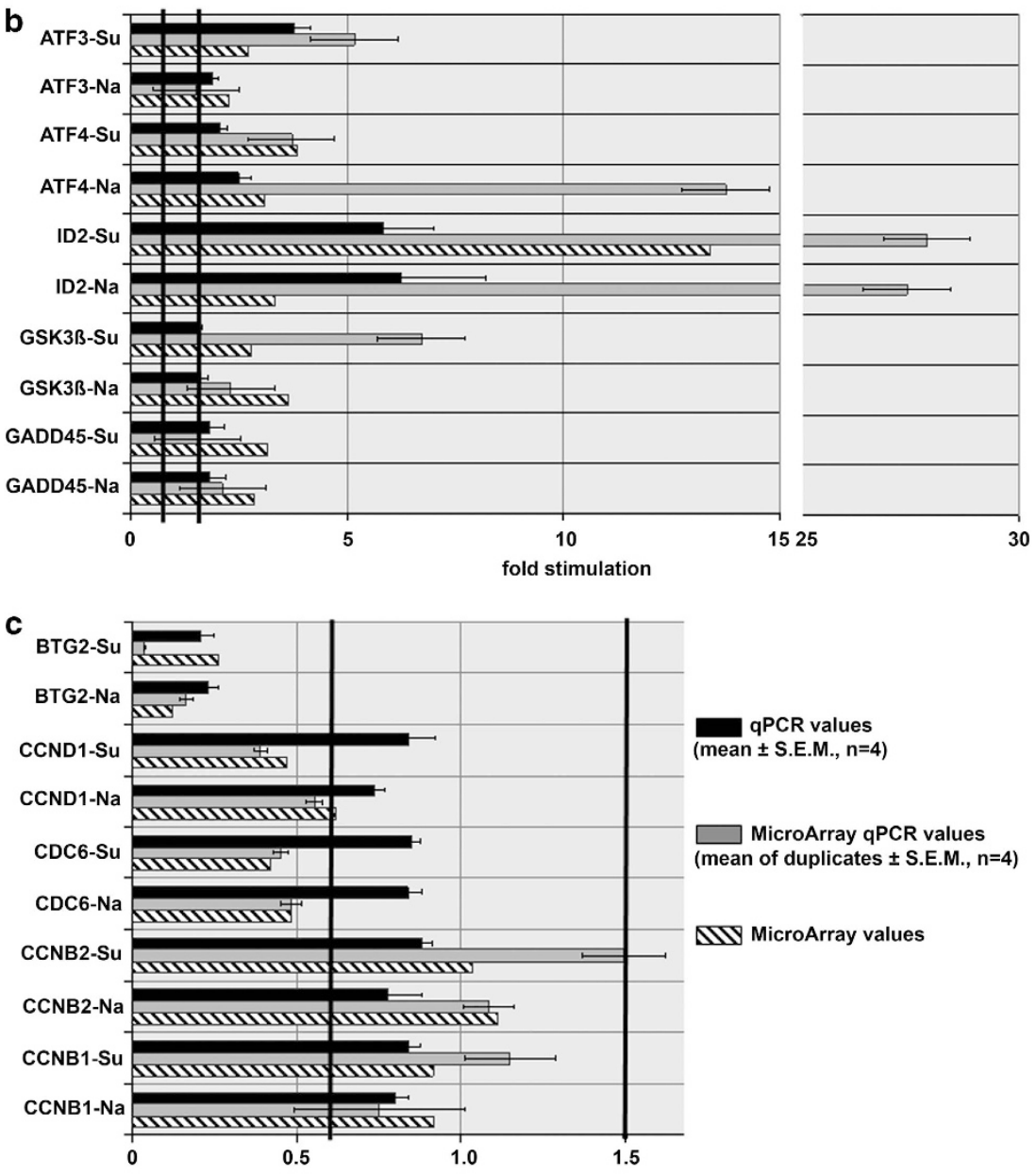

fold stimulation

Figure 1 Validation of microarray data by RT-qPCR methodology. (a) The numbers of overlapping genes modulated in response to hyperosmotic treatments are shown in a Venn diagram. $\uparrow$ represents upregulated genes; $\downarrow$ represents downregulated genes. (b and $\mathbf{c}$ ) Comparison of gene expression data obtained from microarray analysis (gray column; mean of duplicates \pm S.E.M.) and from RT-qPCR analysis performed on samples used for microarray analysis (hashed columns), and on samples obtained from four additional independent experiments (black columns; mean \pm S.E.M., $n=4$ ). The results are expressed as fold stimulation of treated cells over control

and yielded three gene ontology categories significantly modulated (with a false discovery rate, FDR $\leqslant 0.050$ ): regulation of cell proliferation $(F D R=0.017)$, regulation of transcription from RNA polymerase II promoter (FDR $=$ $0.026)$ and response to abiotic stimulus $(F D R=0.042)$. The genes belonging to these categories are listed in Table 1.

Validation of microarray data. Real-time quantitative PCR (RT-qPCR) was performed to validate 13 genes of interest that are involved in cell proliferation, among which several were significantly deregulated in the microarray analysis and others were not deregulated (used as negative controls). The characteristics of 10 selected genes are indicated in Table 2. RT-qPCR was performed on the samples used for microarray analysis as well as on samples generated from four additional independent experiments. Gene expression data obtained by RT-qPCR were compared with those obtained from the microarray analysis (Figure 1). Five genes (ATF3, ATF4, ID2, GSK3 $\beta$ and GADD45) were significantly upregulated and one gene (BTG2) was significantly downregulated in microarray analysis (Figure 1b), and in RT-qPCR performed using the microarray samples (Figure 1b) and RT-qPCR using four independent experiments (Figure 1b). Two genes downregulated in the microarray analysis (CCNB1 and CCNB2) were not deregulated in RT-qPCR performed using the microarray samples (Figure 1c, gray columns) and RT-qPCR using four independent experiments (Figure 1c, black columns). Two genes (CCND1 and CDC6) were significantly downregulated in the microarray analysis and in $\mathrm{RT}-\mathrm{qPCR}$ 
performed using the microarray samples, but non-significantly downregulated in RT-qPCR using four independent experiments (Figure 1c).

Effect of osmotic stress on ARPE-19 cell number, cell proliferation and cell cycle phases. As genes involved in

Table 1 List of genes belonging to the three gene ontology categories displaying a FDR inferior to $5 \%$, following functional annotation chart analysis using DAVID bioinformatics resources

ADAMTS1, ADAM metallopeptidase with thrombospondin type 1 motif, 1

ADRB2, adrenergic beta-2-receptor, surface

ATF3, activating transcription factor 3

ATF4, activating transcription factor 4 (tax-responsive enhancer element B67); activating transcription factor 4C

BCL6, B-cell CLL/lymphoma 6

BCOR, BCL6 corepressor

BMP4, bone morphogenetic protein 4

BRCA1, breast cancer 1 , early onset

BTG2, BTG family, member 2

CBX4, chromobox homolog 4 (Pc class homolog, Drosophila)

CDC6, cell division cycle 6 homolog (S. cerevisiae)

FOSL1, FOS-like antigen 1

HBEGF, heparin-binding EGF-like growth factor

HSPB8, heat shock $22 \mathrm{kDa}$ protein 8

ID2, inhibitor of DNA-binding 2, dominant-negative helix-loop-helix protein

IVL, involucrin

LIF, leukemia inhibitory factor (cholinergic differentiation factor)

MAT2A, methionine adenosyltransferase II, alpha

NEK1 NIMA, (never in mitosis gene a)-related kinase 1

NFKBIA, nuclear factor of kappa light polypeptide gene enhancer in B-cells inhibitor, alpha

PPM1D, protein phosphatase 1D magnesium-dependent, delta isoform

Rho, rhodopsin

RYBP, RING1- and YY1-binding protein

SMAD3, SMAD family member 3

SMAD7, SMAD family member 7

TGM2, transglutaminase 2 (C polypeptide, protein-glutaminegamma-glutamyltransferase)

TXNIP, thioredoxin-interacting protein

VHL, von Hippel-Lindau tumor suppressor

YY1, YY1 transcription factor

ZNF281, zinc-finger protein 281 cell proliferation were significantly modulated, we explored further the effects of osmotic stress on RPE cell proliferation. In response to 24-h osmotic stress, ARPE-19 cell number decreased significantly compared with unstimulated cells (Figure 2a). Apoptosis and cell death did not account for this effect, as no statistically significant modification in the percentage of normal, early apoptotic, late apoptotic and necrotic cells was found following $24 \mathrm{~h}$ osmotic stress when compared with control condition (Table 3). However, following osmotic stress, quantification of cell cycle phases
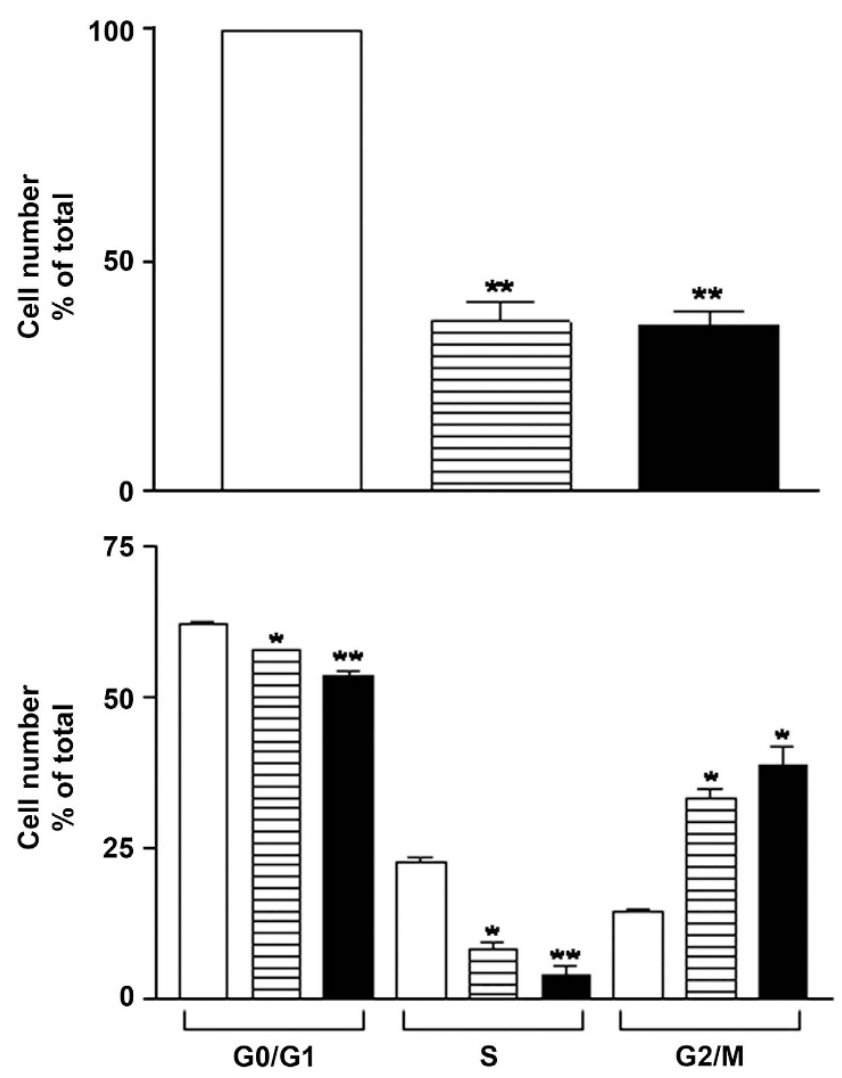

Figure 2 Effect of hyperosmotic stimulation on ARPE-19 cell number and cell cycle phases. ARPE-19 cells were submitted to 24-h stimulation without (open column) or with $100 \mathrm{mM} \mathrm{NaCl}$ (hatched column) or $200 \mathrm{mM}$ sucrose (filled column). (a) Cells were counted in triplicate. (b) Cells were submitted to $\mathrm{PI}$ and BrdU staining and analyzed by FACS as described in Materials and Methods. Results are expressed as cell number (in percent of control) and are the mean \pm S.E.M. $(n=3)$. Data were analyzed using repeated-measures ANOVA and Tukey's multiple comparison tests. ${ }^{*} P<0.01 ;{ }^{* *} P<0.001$ compared with control

Table 2 Gene description

\begin{tabular}{lll}
\hline Gene name & GenBank accession no. & Description \\
\hline hs-ATF4 & NM_001675.2 & Activating transcription factor 4 (tax-responsive enhancer element B67) \\
hs-ATF3 & NM_001030287.3 & Activating transcription factor 3 \\
hs-BTG2 & NM_006763.2 & BTG family, member 2 (NGF-inducible antiproliferative protein PC3) \\
hs-CCNB1 & NM_031966.3 & Cyclin B1 \\
hs-CCNB2 & NM_004701.3 & Cyclin B2 \\
hs-CCND1 & NM_053056.2 & Cyclin D1 \\
$h s-C D C 6$ & NM_001254.3 & Cell division cycle 6 homolog (S. cerevisiae) \\
$h s-G A D D 45 a$ & NM_001924.3 & Growth arrest and DNA-damage-inducible, alpha \\
$h s-G S K 3 b e t a$ & NM_001146156 & Glycogen synthase kinase 3 beta \\
$h s-I D 2$ & NM_002166.4 & Inhibitor of DNA-binding 2, dominant-negative helix-loop-helix protein
\end{tabular}


revealed a decrease in the percentage of cells in G0/G1 and $S$ phases, and an increase in the percentage of cells in $G_{2} / M$ phases (Figure 2b).

Cell cycle protein expression following osmotic stress. As ARPE-19 cell proliferation and the modification of cell cycle phases in response to hyperosmotic stress were inhibited, the expression of various cell cycle proteins was studied after $4 \mathrm{~h}$ and $24 \mathrm{~h}$ of osmotic stress (Figure 3). Cyclin D1 protein levels were decreased at $4 \mathrm{~h}$ upon osmotic stress induced by $\mathrm{Na100}$ or Su200. The effect of osmotic stress on cyclin D1 expression is even more pronounced when cells are exposed to osmotic stress for $24 \mathrm{~h}$, and where even the complete disappearance of cyclin D1 was observed upon osmotic stress under sucrose. (Figure 3a). Cyclin B1 expression seems to be unaffected when cells are exposed to osmotic stress for $4 \mathrm{~h}$; in contrast, after $24 \mathrm{~h}$ exposure to osmotic stress cyclin B1 underwent a strong suppression of its expression level (Figure 3a). The expression of cyclin A1

Table 3 Lack of hyperosmotic stress on ARPE-19 cell death.

\begin{tabular}{lccrr}
\hline & N & EA & LA & \multicolumn{1}{c}{ NC } \\
\hline Control & $95.13 \pm 0.38$ & $0.04 \pm 0.02$ & $0.11 \pm 0.02$ & $4.71 \pm 0.37$ \\
Na100 & $90.88 \pm 2.70$ & $0.02 \pm 0.01$ & $0.05 \pm 0.04$ & $9.05 \pm 2.67$ \\
Su200 & $89.14 \pm 5.02$ & $0.04 \pm 0.01$ & $0.13 \pm 0.05$ & $10.69 \pm 4.98$
\end{tabular}

ARPE-19 cells were submitted to 24-h stimulation without (Control) or with $100 \mathrm{mM} \mathrm{NaCl}$ (Na100) or $200 \mathrm{mM}$ sucrose (Su200). Cells stained with propidium iodide and annexin IV were analyzed by FACS. Results are expressed as normal (N), early apoptotic (EA), late apoptotic (LA) or necrotic (NC) cells (in percent of total), and are the mean \pm S.E.M. $(n=3)$. Data were analyzed using repeated-measures ANOVA and Tukey's multiple comparison tests, $P>0.05$

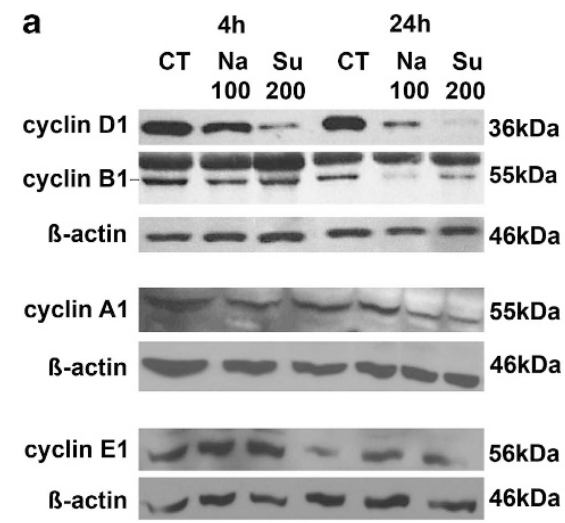

C
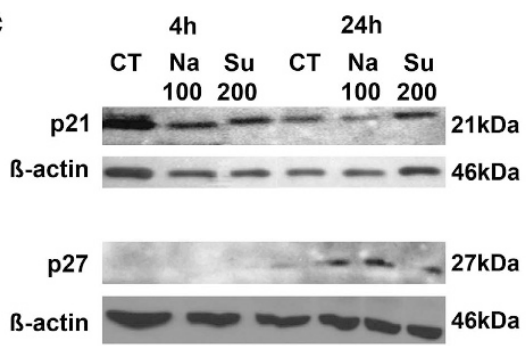
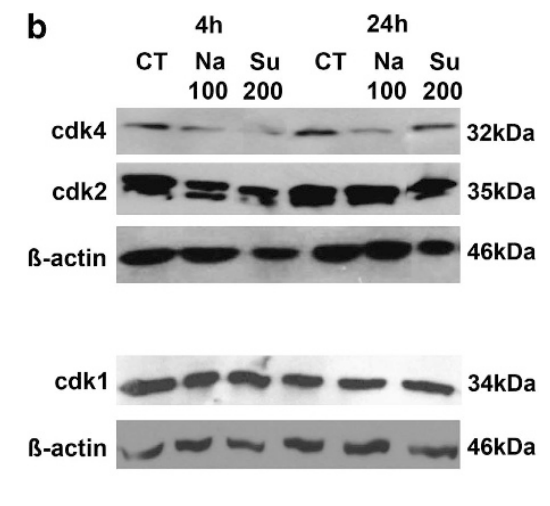

was slightly reduced following $24 \mathrm{~h}$ exposure to osmotic stress in our experiments. The expression of cyclin E1 was slightly increased following $24 \mathrm{~h}$ exposure to osmotic stress. Cyclin-dependent kinase (cdk) 4 expression levels seemed to be slightly diminished upon treatment of cells with sucrose for $4 \mathrm{~h}$ and $\mathrm{NaCl}$ for $24 \mathrm{~h}$ (Figure $3 \mathrm{~b}$ ). The protein expression levels of cdk1 and cdk2 were unaltered upon exposure of ARPE-19 cells to hyperosmotic stress (Figure $3 b$ ). We show that the protein expression level of the cell cycle inhibitor p21 was relatively stable in ARPE-19 cells exposed to osmotic stress. We detected a slight upregulation of cell cycle inhibitor p27 following 4-h stimulation with $\mathrm{NaCl}$, which remains relatively stably expressed following 24-h stimulation by both $\mathrm{NaCl}$ or sucrose. In order to check the expression levels of protein involved in DNA replication and DNA repair, we tested the expression levels of CDC6 and proliferating cell nuclear antigen (PCNA). Upon hyperosmotic stress of ARPE-19 cells, the protein expression levels of PCNA and CDC6 were not modified (Figure 3d).

The involvement of the mitogen-activated protein kinase (MAPK) pathway in the osmotic stress response of ARPE-19 cells (at 4 and $24 \mathrm{~h}$ ) was also investigated by the detection of p38 activation using an anti-phospho p38 antibody, specifically recognizing the active double-phosphorylated protein. Indeed, phosphorylated p38 was detected at a low level following $4 \mathrm{~h}$ of Su200 treatment, and at a higher level following $24 \mathrm{~h}$ of Na100 or Su200 treatments (Figure 4a). Furthermore, $1 \mu \mathrm{M}$ SB203580, a p38 inhibitor, decreased the expression of cyclin B1 at $24 \mathrm{~h}$ post-hypertonic treatment with Na100 or Su200, whereas $10 \mu \mathrm{g} / \mathrm{ml}$ anisomycine, a p38 activator, decreased cyclin B1 expression at 4 and $24 \mathrm{~h}$ after incubation compared with control conditions (Figure 4b).
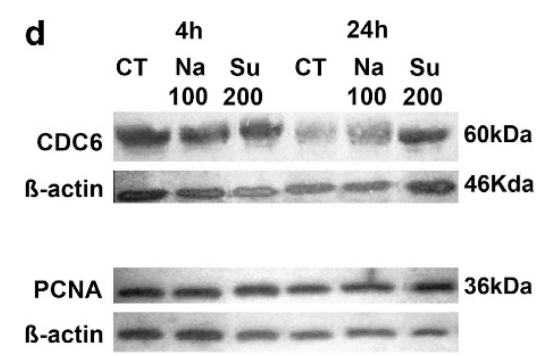

Figure 3 Cyclins and cdks expression during hyperosmotic stress. (a-e) ARPE-19 cells were submitted to 4- or 24-h stimulation without (Ct) or with $100 \mathrm{mM} \mathrm{NaCl}$ (Na100) or $200 \mathrm{mM}$ sucrose (Su200). Total protein expression of cyclins D1, B1, A1, E1 and $\beta$-actin (a); cdk1, cdk2, cdk4 and $\beta$-actin (b); p21, p27 and $\beta$-actin (c); CDC6, PCNA and $\beta$-actin (d); were determined by western blot analysis 

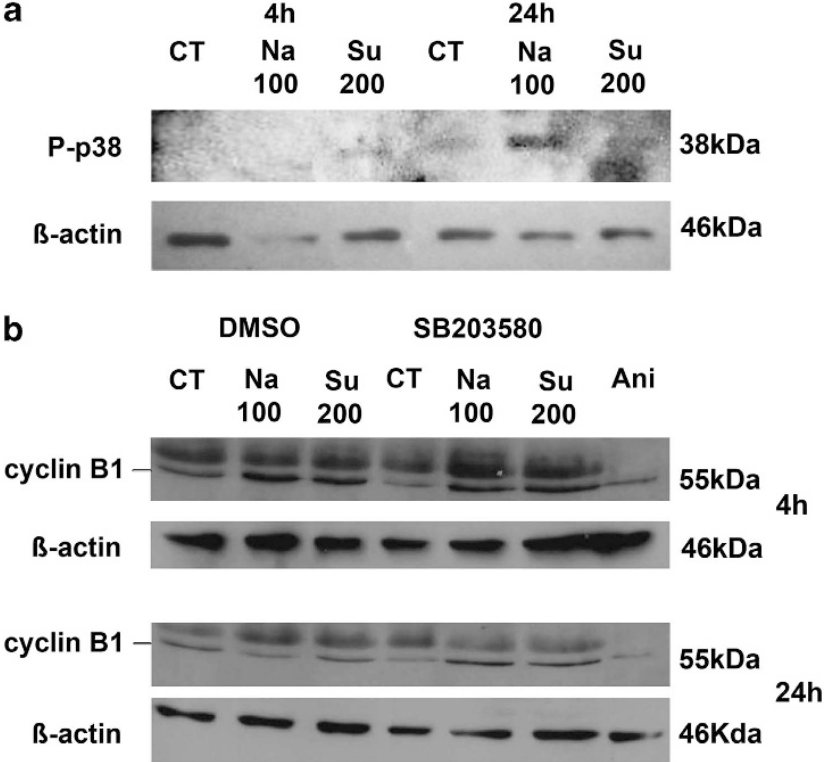

Figure 4 Involvement of p38 during hyperosmotic stress. (a) Phosphorylated p38 was determined by western blot in ARPE-19 cells submitted to 4- or 24-h stimulation without (Ct) or with $100 \mathrm{mM} \mathrm{NaCl}$ (Na100) or $200 \mathrm{mM}$ sucrose (Su200). (b) Cyclin B1 expression was determined by western blot in ARPE-19 cells submitted to 1 -h preincubation without (DMSO) or with $1 \mu \mathrm{M}$ SB203580, followed by a 4- or 24-h stimulation without (Ct) or with Na100 or Su200, as well as to 4- or 24-h incubation with $10 \mu \mathrm{g} / \mathrm{ml}$ anisomycin (Ani)

\section{Discussion}

Despite possible extreme hyperosmotic conditions, cells can survive and function owing to protective adaptations including accumulation of large amounts of organic osmolytes, which normalize cell volume and intracellular ionic strength. In RPE cells, it has been shown that hyperosmolarity induces specific cellular responses, such as the specific increase of the transcription factor NFAT5, which in turn regulates, amongst others, aldose reductase activity, and taurine transporter expression and function. ${ }^{3,5}$ However, it has been described that osmostress response can have a wide impact on a variety of cell type behavior, and modulates the expression and activity of many proteins.

Our data support the hypothesis that hyperosmotic stress can largely modulate non-kidney-type cell functions, as we found that upon $4 \mathrm{~h}$ exposure to $\mathrm{NaCl} 100 \mathrm{mM}$ or sucrose $200 \mathrm{mM}$ the expression of 151 genes were similarly altered, with 79 downregulated and 72 upregulated. Three gene ontology categories were significantly modulated: regulation of cell proliferation, regulation of transcription from RNA polymerase II promoter and response to abiotic stimulus. The finding that many genes involved in cell proliferation were regulated in ARPE-19 cells was highly consistent with the notion that during early osmoadaptation, cells induce an elaborate program that includes changes in transcription and translation, as well as cell cycle progression to allow cells to adapt. Our cell cycle analysis supported this further. Indeed, we found that in ARPE-19 cells submitted to hyperosmotic stress, cells accumulate in $\mathrm{G} 1 / \mathrm{S}$ and $\mathrm{G}_{2} / \mathrm{M}$ phases and incorporated less efficiently bromo-deoxyuridine (BrdU), accounting for cell cycle arrest and consequently leading to decreased cell proliferation.

Cell cycle progression is regulated by sequential activation of cdks and their association with cyclins. ${ }^{6}$ The cyclin D/cdk4 complex formation ensures cell cycle progression into the $\mathrm{G} 1$ phase, whereas the cyclin E/cdk2 complex formation is required for the $\mathrm{G} 1 / \mathrm{S}$ transition. The cyclin $\mathrm{A} / \mathrm{cdk} 2$ complex formation drives cell cycle progression into $S$ phase, whereas the cyclin A/cdk1 complex drives G2 phase. Finally, the cyclin $\mathrm{B} / \mathrm{cdk} 1$ formation leads to G2/M transition. Cdks inhibitors compete or cooperate with cyclins for their binding to cdks. ${ }^{7,8,9}$ The functions of PCNA include its ability to act as DNA sliding clamp for replicative DNA polymerases, its involvement in DNA replication and repair, its ability to stimulate the activity of DNA polymerase $\delta$, and its ability to interact with cyclin/cdk complexes and p21. ${ }^{10-11}$ Binding of PCNA to cyclin/cdk complexes might help them to reach their targets, whereas disruption of these interactions by the competitive binding of p21 is a signal for cell cycle arrest. ${ }^{11}$ CDC6 is an essential regulator of DNA replication as it drives the assembly of the pre-replicative complexes at origins of replication during the $\mathrm{G} 1$ phase of the cell cycle division. ${ }^{12}$ Besides, CDC6 has a crucial role in the activation and maintenance of the checkpoints' mechanisms coordinating $S$ phase and mitosis. ${ }^{12,13}$ In a situation of cellular stress, CDC6 is at the crossroads of two seemingly opposing pathways, one that stabilizes pre-replicative complexes in order to activate the G1/S and G2/M checkpoint signaling, and another one that destabilizes them in order to avoid further initiation events at replication origins.

The observed cell cycle arrest was corroborated by the decreased expression of cyclins D1 and B1, and increased p27 expression. The expression of different cdks, like G1-regulating cdk4, S-phase cdk2 and mitotic cdk1, were slightly decreased (cdk4) or unaltered by hyperosmotic stress (cdk2 and cdk1). It is known that cdk activity can be largely regulated by the presence or the absence of their cyclin partners. ${ }^{14}$ Thus, downregulation of cyclin D1 and cyclin B1 contributes to the inactivation of cdk4 and cdk1, respectively, which leads to cell cycle arrest of ARPE-19 cells.

Furthermore, steady expression levels of p21 might contribute to their interaction with PCNA and block both its activity necessary for DNA replication and its binding to cdk/ cyclin complexes necessary to bring these regulatory proteins to their targets. ${ }^{11,15}$ The action of p21 could thus consequently lead to the arrest of the cell cycle at the G1/S and G2/M checkpoints. The upregulation of Gadd45, observed only at the mRNA level (as the antibodies used against Gadd45 were not efficient in our hands), might also participate to $\mathrm{G} 1$ arrest by interacting with $\mathrm{p} 21,{ }^{16,17,18}$ and to $\mathrm{G} 2$ arrest by cdk1/ cyclin B1 complex, resulting in both cases in decreased cdk1 activity. ${ }^{19}$ As CDC6 expression was not modified following osmotic stress, it may not be directly involved in the observed cell cycle arrest.

Cell exposure to hyperosmotic medium resulted in rapid activation of $\mathrm{G} 1 / \mathrm{S}$ and $\mathrm{G}_{2} / \mathrm{M}$ checkpoints. Activation of $\mathrm{G} 1$ and $S$ checkpoints depends on tumor protein 53 activity, but activation of $G_{2} / M$ checkpoint is induced by stressactivated MAPK. ${ }^{20}$ That osmotic stress activates MAPKs was first described in Saccharomyces cerevisiae and later 
Table 4 RT-qPCR primers

\begin{tabular}{|c|c|c|c|}
\hline Gene name & Forward primer sequence & Reverse primer sequence & Efficiency (\%) \\
\hline hs-ATF4 & 5'-CCAACAACAGCAAGGAGGATG-3' & 5'-CCAACGTGGTCAGAAGGTCA-3' & 95 \\
\hline hs-ATF3 & 5'-TGTCCATCACAAAAGCCGAG-3' & 5'-GACTCTTTCTGCAGGCACTC-3' & 92 \\
\hline hs-BTG2 & 5'-GCTGCCATGATCCCTTTGAG-3' & 5'-CGGTAGCTCTATGACCCGAC-3' & 95 \\
\hline hs-CCNB1 & 5'-AAACTTTCGCCTGAGCCTATT-3' & 5'-CATCAGAGAAAGCCTGACACA-3' & 105 \\
\hline hs-CCNB2 & 5'-CGCATGCGTGCCATCCTAGTGG-3' & 5'-AGCTTCTTCCGGGAAACTGGCTGA-3' & 96 \\
\hline hs-CCND1 & 5'-TCCAGAGTGATCAAGTGTGAC-3' & 5'-GGTCCATGTTCTGCTGGG-3' & 101 \\
\hline hs-CDC6 & 5'-GGATGTTTGCAGGAGAGCTA-3' & 5'-CAGAGGCTCAGAAGGTGATT-3' & 91 \\
\hline hs-GADD45 a & 5'-AGACCGAAAGGATGGATAAGG-3' & 5'-CAGGCACAACACCACGTTA-3' & 91 \\
\hline hs-GSK3beta & 5'-GTCCTGGGAACTCCAACAAG-3' & 5'-GAGTTCGGGGTCGGAAGA-3' & 99 \\
\hline hs-ID2 & 5'-CACGGATATCAGCATCCTGTCC-3' & 5'-ATGAACACCGCTTATTCAGCC-3' & 101 \\
\hline
\end{tabular}

also in mammalian cells. ${ }^{21,22}$ The p38 MAPKs are crucial for both early response and long-term cell adaptation to osmotic stress. $^{1,23}$ It has been shown that p38 activation can affect cell cycle G2/M checkpoint upon hyperosmotic stress. ${ }^{24,25}$ Several MAPKs have been shown recently to be implicated in the $G_{2} / M$ transition, independent of the ATM kinase activation mediating DNA damage repair. ${ }^{26,27}$ In particular, p38 and c-Jun $\mathrm{N}$-terminal kinases are reported to delay progression through $\mathrm{G}_{2}$ in response to osmotic stress, and this effect can be overridden by inhibiting p38 kinase. ${ }^{24,25}$ The association of Gadd45 with p38 kinase has been shown to result in its activation. ${ }^{19}$ In ARPE-19 cells submitted to hyperosmotic stress, p38 kinase was indeed activated in agreement with MAPKs activation induced by osmostress and/or binding with Gadd45. Furthermore, we showed that the activation of p38 following hyperosmotic stress in ARPE-19 cells induced a decrease in cyclin B1 expression that would explain the observed cell cycle arrest under such experimental conditions.

In conclusion, our results demonstrate that osmotic stress profoundly affect gene transcription of RPE cells and control cell proliferation by downregulating cyclin D1 and cyclin B1 protein expression. Further studies are required to determine whether hypertonic conditions modulate other RPE cell functions.

\section{Materials and Methods \\ Materials. ARPE-19 cells were purchased from ATCC-LGC Standards (Molsheim, France). Antibodies for cdk4, cdk1, cdk2, cyclin D1, cyclin A, p27 cip1 p27 $7^{\mathrm{kip} 1}$ and cyclin B1 were from Santa Cruz Biotechnology (Santa Cruz, CA, USA). The antibodies for cyclin E1 and anti-phospho p38 were from Cell Signalling, (Danvers, MA, USA). The antibodies for PCNA and CDC6 were from Millipore-Merck (Billerica, MA, USA).}

Cells. ARPE-19 cells were grown in DMEM/HAM-F12 medium containing $10 \%$ fetal calf serum, $100 \mathrm{UI} / \mathrm{ml}$ penicillin, $100 \mu \mathrm{g} / \mathrm{ml}$ streptomycin and $4 \mathrm{mM}$ glutamine, and passaged twice a week. Cells were treated for 4 or $24 \mathrm{~h}$ without (control) or with additional Na100, or Su200.

Microarray. Total RNA extraction from ARPE-19 cells was carried out using the mirVana miRNA Isolation Kit (Ambion; Austin, TX, USA) according to the manufacturer's instructions. RNA concentration and purity were determined using a NanoDrop 8000 Spectrophotometer (Thermo Scientific, Schwerte, Germany) and RNA integrity was verified on an Experion Bioanalyzer (Bio-Rad Laboratories, Hercules, CA, USA), as previously described. ${ }^{28}$ Double-stranded CDNA was synthesized from $1 \mu \mathrm{g}$ of total RNA, followed by production of antisense RNA containing modified 5-(3-aminoally)-UTP, using the Amino Allyl MessageAmp II aRNA Amplification kit (Ambion). After labeling with Cy3 or Cy5 (GE Healthcare Bio-Sciences, Piscataway, NJ, USA), the samples were hybridized for $16-18 \mathrm{~h}$ on
Human Exonic Evidenced-Based Oligonucleotides slides (Stanford Functional Genomics Facility, Stanford, CA, USA). The oligonucleotide set consists of 49958 different 70-mer probes that were designed using transcriptome-based annotation of exonic structure for genomic loci. Hybridizations were replicated by dye swap. Slides were scanned using a Molecular Device 4000B Laser scanner and expression levels were quantified with GenePix Pro 6.1 image analysis software (Axon Instruments, Sunnyvale, CA, USA). Data were normalized and expressed as the $\log _{2}$ ratio of fluorescence intensities of the sample and the control condition for each spot on the array, and dye swaps were averaged. Spots with missing values for $>20 \%$ of the samples were discarded (13231 remaining genes). The subsets of spots that varied from baseline by at least one-fold in at least one of the hyperosmotic conditions were then selected (2768 genes). Duplicate spots were eliminated (2316 remaining genes). The subset of genes that varied in similar fashion following treatments with Na100 and Su200 was selected (151 genes). This subset of genes was analyzed for global function using DAVID bioinformatic resources 6.7 (NIAID-NIH, MD, U.S.A.) based on Gene Ontology terms enrichment and gene functional classification. ${ }^{29,30}$

Primer design. Primers were designed as previously described ${ }^{28}$ to ensure optimal DNA polymerization efficiency and amplification specificity, as well as optimal amplicon length (100-180bp; Table 4). Primers were mandatorily separated by at least one intron (whenever possible) to minimize amplification from any contaminating genomic DNA that may remain after the RNA purification procedure. Primer pair efficiency was tested by performing a RT-qPCR using between standard curves ranging from $10 \mathrm{ng}$ down to $0.039 \mathrm{ng}$ (four-fold serial dilutions) of an appropriate cDNA template. Possible genomic DNA amplification of primer pairs was verified by performing a RT-qPCR in the presence of $2.5 \mathrm{ng}$ genomic DNA.

Gene expression analysis using quantitative real-time PCR. The modulation of expression of a number of identified modulated genes by microarray analysis was investigated using RT-qPCR in the cell samples used for the microarray analysis (for validation of microarray gene expression data), as well as in the cell samples from four other independent experiments. The RT-qPCR reaction was performed as previously described, using $2.5 \mathrm{ng} \mathrm{CDNA}$ as template using SyberGreen master mix from Eurogentec (WOUB MESA BLUE qPCR Master Mix PI.SYBR Low Rox, Eurogentec, Seraing, Belgium). ${ }^{28}$ Raw data were analyzed for experimental conformity using the StepOne Software version 2.1 (Applied Biosytems, Carlsbad, CA, USA), and data normalization using selected appropriate stable reference genes, and normalized gene expression were obtained through the Biogazelle qBase Plus software Package (Gent, Belgium). ${ }^{31}$ All RT-qPCR procedures were in compliance with the MIQE guidelines. ${ }^{32}$

Protein preparation and western blot analysis. ARPE-19 cells were harvested in lysis buffer (50 mM Tris- $\mathrm{HCl}(\mathrm{pH} 7.5), 150 \mathrm{mM} \mathrm{NaCl}, 0.5 \% \mathrm{NP}-40)$, containing $50 \mathrm{mM}$ sodium fluoride, $1 \mathrm{mM}$ sodium orthovanadate and a cocktail of protease inhibitors (Complete EDTA free, Roche Applied Science, Mannheim, Germany). Harvested ARPE-19 cells were immediately frozen in liquid nitrogen, then thawed and submitted to a 10 -min centrifugation at $15000 \times g$ at $4^{\circ} \mathrm{C}$. The supernatant containing total proteins was collected. Protein concentration was determined using a BCA protein assay kit (Pierce, Rockford, IL, USA). Total proteins, in the presence of $5 \% \beta$-mercaptoethanol, were analyzed by SDS-PAGE. Separated proteins were transferred to PVDF membranes and immunolabeled 
using specific primary antibodies. The bound primary antibodies were detected using secondary anti-mouse or anti-rabbit antibodies (GE Healthcare, Buckinghamshire, UK) and ECL chemiluminescence detection kit (Perkin Elmer, Waltham, MA, USA).

Cell count. ARPE-19 cells, grown in six-well plates, were trypsinized, centrifuged for $5 \mathrm{~min}$ at $500 \times g$, resuspended into Coulter Isoton II diluent and counted using a Coulter Multisizer II (Beckman Coulter, Inc., Miami, FL, USA).

FACS analysis. The fluorescence of different markers was analyzed by using fluorescent-activated cell sorting (FACS) analysis. For propidium iodide (PI) labeling, subconfluent ARPE-19 cells were trypsinized, fixed with ice-cold $70 \%$ ethanol for $30 \mathrm{~min}$ on ice, incubated at room temperature $\left(2 \times 10^{6}\right.$ cells $\left./ \mathrm{ml}\right)$ in PBS with $0.1 \mathrm{mg} / \mathrm{ml}$ RNAse for $15 \mathrm{~min}$, and then with $100 \mu \mathrm{g} / \mathrm{ml}$ of PI for $20 \mathrm{~min}$, and analyzed on a FACSscan instrument (FACS-Scan, Becton Dickinson, San Jose, CA, USA). For double labeling with annexin-V-FITC and $\mathrm{PI}$, subconfluent ARPE-19 cells were trypsinized, incubated with annexin-V-FITC for $15 \mathrm{~min}$ at room temperature, and then with $50 \mu \mathrm{g} / \mathrm{ml}$ of $\mathrm{PI}$ for an additional $15 \mathrm{~min}$ at room temperature, washed and resuspended in PBS before FACS analysis. For double labeling with anti-BrdU and PI, subconfluent ARPE-19 cells were trypsinized following a 30-min pulse with $10 \mu \mathrm{M}$ BrdU, washed, fixed with ice-cold $70 \%$ ethanol for $30 \mathrm{~min}$ on ice, washed, incubated in $2 \mathrm{~N} \mathrm{HCl}$ with $0.5 \%$ Tween-20 for $30 \mathrm{~min}$ at room temperature, washed, and then subsequently incubated for additional 30 min without or with mouse anti-BrdU antibodies (1: 10; Roche Applied Science), washed, and then incubated for 30 min without or with anti-mouse-fluorescein (1:10; Roche Applied Science). Finally, the cells were incubated in PBS with $0.1 \mathrm{mg} / \mathrm{ml}$ RNAse for $15 \mathrm{~min}$, then with $100 \mu \mathrm{g} / \mathrm{ml}$ of PI for $20 \mathrm{~min}$, washed, and then analyzed on a FACSscan instrument. The data were analyzed using the CellQuest analysis software (BD Biosciences, Franklin lakes, NJ, USA).

Statistical analysis. Statistical analyses were performed using repeated measures ANOVA and Tukey's multiple comparison tests or paired Student $t$-tests.

\section{Conflict of Interest}

The authors declare no conflict of interest.

Acknowledgements. This work was supported by Grant 3.4502 .09 from the Fund for Medical Scientific Research (FRSM, Belgium) and from the Funds for Research in Ophthalmology (FRO, Belgium). TA is a recipient of a FNRS fellowship (FNRS, Belgium), SJ is a recipient of a Vésale Foundation Award.

\section{Author contributions}

$\mathrm{TA}, \mathrm{AOdB}, \mathrm{JP}, \mathrm{FL}, \mathrm{AL}, \mathrm{FW}$ and $\mathrm{CD}$ conceived and/or designed the work; $\mathrm{TA}$ $A V, A O d B, F L, S J, A L, F G, N B$ and $C D$ acquired data; $T A, A V, A O d B, F L, A H, A L$ $J P, F W$ and $C D$ analyzed and interpreted data; TA, AV, $A O d B, J P, F W$ and $\mathrm{CD}$ wrote the paper. TA, AV, FL, AOdB, AH, SJ, FG, AL, NB, JP, LC, FW and CD approved the final version.

1. Burg MB, Ferraris JD, Dmitrieva NI. Cellular response to hyperosmotic stresses. Physiol Rev 2007; 87: 1441-1474.

2. Brocker $\mathrm{C}$, Thompson DC, Vasiliou V. The role of hyperosmotic stress in inflammation and disease. Biomol Concepts 2012; 3: 345-364.

3. Lin LR, Carper D, Yokoyama T, Reddy VN. The effect of hypertonicity on aldose reductase, alpha B-crystallin, and organic osmolytes in the retinal pigment epithelium. Invest Ophthalmol Vis Sci 1993; 34: 2352-2359.

4. Strauss 0 . The retinal pigment epithelium in visual function. Physiol Rev 2005; 85 : 845-881.

5. El Sherbeny A, Naggar $\mathrm{H}$, Miyauchi S, Ola MS, Maddox DM, Martin PM et al. Osmoregulation of taurine transporter function and expression in retinal pigment epithelial ganglion, and muller cells. Invest Ophthalmol Vis Sci 2004; 45: 694-701.
6. Xiong Y, Connolly T, Futcher B, Beach D. Human D-type cyclin. Cell 1991; 65: 691-699

7. Takahashi H, Menjo M, Kaneko Y, Ikeda K, Matsushime H, Nakanishi M. Cdk4 activation is dependent on the subunit rearrangement in the complexes. Biochem Biophys Res Commun 2000; 267: 388-393.

8. Bockstaele L, Kooken H, Libert F, Paternot S, Dumont JE, de Launoit $Y$ et al. Regulated activating Thr172 phosphorylation of cyclin-dependent kinase 4 (CDK4): its relationship with cyclins and CDK 'inhibitors'. Mol Cell Biol 2006; 26: 5070-5085.

9. Coulonval K, Bockstaele L, Paternot S, Dumont JE, Roger P. The cyclin D3-CDK4-p27kip1 holoenzyme in thyroid epithelial cells: activation by TSH, inhibition by TGFbeta, and phosphorylations of its subunits demonstrated by two-dimensional gel electrophoresis. Exp Cell Res 2003; 291: 135-149.

10. Strzalka W, Ziemienowicz A. Proliferating cell nuclear antigen (PCNA): a key factor in DNA replication and cell cycle regulation. Ann Bot 2011; 107: 1127-1140.

11. Maga G, Hubscher U. Proliferating cell nuclear antigen (PCNA): a dancer with many partners. J Cell Sci 2003; 116: 3051-3060

12. Borlado MR, Méndez J. CDC6: from DNA replication to cell cycle checkpoints and oncogenesis. Carcinogenesis 2008; 29: 237-243.

13. Boronat S, Campbell JL. Linking mitosis with S-phase: Cdc6 at play. Cell Cycle 2008; 7: $597-601$.

14. Sherr CJ. Mammalian G1 cylins and cell cycle progression. Proc Assoc Am Phys 1995; 107: $181-186$.

15. Tsurimoto T. PCNA binding proteins. Front Sci 1999; 4: D849-D859.

16. Wang XW, Zhan Q, Coursen JD, Khan MA, Kontny HU, Yu L et al. GADD45 induction of a G2/M cell cycle checkpoint. Proc Natl Acad Sci USA 1999; 96: 3706-3711.

17. Vairapandi M, Balliet AG, Hoffman B, GADD45b Liebermann DA. and GADD45g are cdc2/ cyclinB1 kinase inhibitors with a role in $\mathrm{S}$ and $\mathrm{G} 2 / \mathrm{M}$ cell cycle checkpoints induced by genotoxic stress. J Cell Physiol 2002; 192: 327-338.

18. Jin S, Antinore MJ, Lung FD, Dong X, Zhao H, Fan F et al. Roller PP, Fornace Jr AJ, Zhan $Q$. The GADD45 inhibition of Cdc2 kinase correlates with GADD45-mediated growth suppression. J Biol Chem 2000; 275: 16602-16608.

19. Liebermann DA, Hoffman B. Gadd45 in stress signaling. J Mol Signal 2008; 3.

20. de Nadal E, Alepuz PM, Posas F. Dealing with osmostress through MAP kinase activation. EMBO Rep 2002; 3: 735-740.

21. Hohmann S. Osmotic stress signaling and osmoadaptation in yeasts. Microbiol Mol Biol Rev 2002; 66: 300-372.

22. Sheikh-Hamad D, Gustin MC. MAP kinases and the adaptative response to hypertonicity: functional preservation from yeasts to mammals. Am J Physiol 2004; 287: F1102-F1110.

23. Cuenda A, Rousseau S. p38 MAP-kinases pathway regulation, function and role in human diseases. Biochim Biophys Acta 2007; 1773: 1358-1375.

24. Dmitrieva NI, Bulavin DV, Fornace AJ, Burg MB. Rapid activation of G2/M checkpoint after hypertonic stress in renal inner medullary epithelial (IME) cells is protective and requires p38 kinase. Proc Natl Acad Sci USA 2002; 99: 184-189.

25. Kultz D. Phylogenetic and functional classification of mitogen- and stress-activated protein kinases. J Mol Evol 1998; 46: 571-588.

26. Bulavin DV, Amundson SA, Fornace AJ. p38 and Chk1 kinases: different conductors for the G2/M checkpoint symphony. Curr Opin Genet Dev 2002; 12: 92-97.

27. Pearce AK, Humphrey TC. Integrating stress-response and cell-cycle checkpoint pathways. Trends Cell Biol 2001; 11: 426-433.

28. Arsenijevic T, Grégoire F, Delforge V, Delporte C, Perret J. Murine 3T3-L1 adipocyte cell differentiation model: validated reference genes for $\mathrm{qPCR}$ gene expression analysis. PLos ONE 2012; 7: e37517.

29. Huang DW, Sherman BT, Lempicki RA. Systematic and integrative analysis of large gene lists using DAVID bioinformatics resources. Nat Protoc 2009; 4: 44-57.

30. Huang DW, Sherman BT, Lempicki RA. Bioinformatics enrichment tools: paths toward the comprehensive functional analysis of large gene lists. Nucleic Acids Res 2009; 37: 1-13.

31. Vandesompele J, De Preter K, Pattyn F, Poppe B, Van Roy N, De Paepe A et al. Accurate normalization of real-time quantitative RT-PCR data by geometric averaging of multiple internal control genes. Genome Biol 2002; 3, RESEARCH0034-1RESEARCH0034-11.

32. Bustin SA. Quantification of mRNA using real-time reverse transcription PCR (RT-PCR): trends and problems. J Mol Endocrinol 2002; 29: 23-39.

(c) (1) () $\odot$ Cell Death and Disease is an open-access journal published by Nature Publishing Group. This work is licensed under a Creative Commons Attribution-NonCommercialNoDerivs 3.0 Unported License. To view a copy of this license, visit http://creativecommons.org/licenses/by-nc-nd/3.0/ 